\title{
Suppression of spontaneous emission for two-dimensional GaAs photonic crystal microcavities
}

Søndergaard, Thomas; Broeng, Jes; Bjarklev, Anders Overgaard

Published in:

Summaries of Papers Presented at the Quantum Electronics and Laser Science Conference, 1999. QELS '99. Technical Digest.

Link to article, DOI:

10.1109/QELS.1999.807632

Publication date:

1999

Document Version

Publisher's PDF, also known as Version of record

Link back to DTU Orbit

Citation (APA):

Søndergaard, T., Broeng, J., \& Bjarklev, A. O. (1999). Suppression of spontaneous emission for twodimensional GaAs photonic crystal microcavities. In Summaries of Papers Presented at the Quantum Electronics and Laser Science Conference, 1999. QELS '99. Technical Digest. IEEE. https://doi.org/10.1109/QELS.1999.807632

\section{General rights}

Copyright and moral rights for the publications made accessible in the public portal are retained by the authors and/or other copyright owners and it is a condition of accessing publications that users recognise and abide by the legal requirements associated with these rights.

- Users may download and print one copy of any publication from the public portal for the purpose of private study or research.

- You may not further distribute the material or use it for any profit-making activity or commercial gain

- You may freely distribute the URL identifying the publication in the public portal 
JFA

8:00 am-10:00 am

Rooms 327/329

\section{Active Photonic Crystals and Microstructures}

TBA, Presider

\section{JFA1 (Invited)}

8:00 am

Strong Purcell effect for quantum boxes in micropillars and microdisks

J.M. Gérard, B. Gayral, B. Sermage, France Télécom/CNET/DTD/CDP, 196 av. $H$. Ravera, F-92225 Bagneux Cedex, France; E-mail: jeanmichel.gerard@cnet.francetelecom.fr In a founding paper, ${ }^{1}$ Purcell proposed to control the spontaneous emission (SE) rate of a dipole by using a cavity to tailor the dipolefield coupling. In spite of a major experimental effort motivated by the interest of this concept in optoelectronics, only minor SE rate enhancements could be observed in solid-state microcavities until recently.

Very severe requirements must be fulfilled by both the cavity and the emitter for observing this effect. According to Purcell, an emitter on resonance with a discrete cavity mode experiences a $\mathrm{SE}$ enhancement of the order of $3 / 4 \pi^{2} \mathrm{Q} \lambda^{3} / \mathrm{V}=\mathrm{Fp}$, where $\mathrm{V}$ is the cavity mode volume, $Q$ the cavity quality factor and $\lambda$ the wavelength in the material, but this result is valid only as long as the emitter linewidth is narrower than the cavity mode. Usual solidstate emitters are quite broad spectrally, which puts a stringent upper limit on Q. Selfassembled InAs/GaAs quantum boxes (QBs) are thus very interesting in this context: isolated QBs display a single sharp $(\sim 50 \mu \mathrm{eV})$ emission line, which allows potentially to implement very high $Q$ semiconductor cavities (up to $Q \sim 20000$ ).

Progress in microfabrication techniques has allowed a $3 \mathrm{D}$ engineering of the refraction index on the wavelength scale and a rich diversification of microcavity designs (micropillars, microdisks, photonic wires, microcavities based on photonic bandgap material ...). When total internal reflection or waveguiding are used to confine light, the optical quality of small volume microcavities is extremely sensitive to the morphology of their sidewalls. The scattering by surface roughness provides an additional escape path to confined photons, which reduces their lifetime in the cavity and $\mathrm{Q}$. We report the fabrication of both GaAs/ AlAs micropillars and GaAs microdisks displaying an improved optical quality. By optimizing the reactive ion etching of micropillars, Q's as high as 2000 for $1 \mu \mathrm{m}$ diameter pillars could be obtained $(\mathrm{Fp}=32)$. Microdisks have been defined through a novel process based on a two-step wet etching. Whispering gallery modes display then record high Q's (12000 for $2 \mu \mathrm{m}$ diameter disks instead of 1500 for the previous state of the art) which highlights a spectacular reduction of the microdisks edge roughness and corresponds to a very high $\mathrm{Fp}$ $(\sim 150)$.
By inserting QBs in such small volume/ high $Q$ microcavities, we could observe for the first time a very strong Purcell effect in the solid-state. ${ }^{2}$ For GaAs/AlAs pillar microcavities, a (up to) five-fold shortening of the radiative lifetime is observed by time-resolved photoluminescence (PL) for the $\mathrm{QBs}$ which are on-resonance with a cavity mode, whereas it is essentially unmodified for off-resonance QBs. A simple model taking into account the spatial/spectral distribution of the QBs in the pillar and its Purcell factor Fp explains quantitatively the magnitude of this SE enhancement. Though our microdisks have not yet been studied by time-resolved PL, a clear signature of the Purcell effect could be observed in a simple c.w. experiment. In our structures, the number of QBs coupled to a given mode is not large enough to sustain lasing. When we raise the excitation power, the emission related to the fundamental optical transition of a QB thus saturates when we inject more than one electron-hole pair per lifetime. This behavior is observed for on-resonance as well as offresonance QBs. However the onset of the saturation occurs for a much higher pumping power when $\mathrm{QBs}$ are resonantly coupled to a whispering gallery mode, which confirms that their radiative lifetime can be (up to) 20 times shorter due to the Purcell effect.

Beyond this result, InAs QBs will probably allow to witness other CQED effects in solidstate microcavities (e.g. the strong coupling regime for a single $\mathrm{QB}$ ) and open unique opportunities for the development of single photon light emitters for applications such as quantum cryptography or metrology.

1. E.M. Purcell, Phys. Rev. 69, 681 (1946).

2. J.M. Gérard et al., Phys. Rev. Lett. 81, 1110 (1998).

\section{JFA2}

8:30 am

Suppression of spontaneous emission for two-dimensional GaAs photonic crystal microcavities

Thomas Søndergaard, Jes Broeng, Anders Bjarklev, Department of Electromagnetic Systems Technical University of Denmark, Building 348, DK-2800 Lyngby, Denmark

Spontaneous emission represents a loss mechanism that fundamentally limits the performance of semiconductor lasers. The rate of spontaneous emission may, however, be controlled by a new class of periodic dielectric structures known as photonic crystals. Although a three-dimensional periodic structure may rigorously forbid spontaneous emission within a frequency interval, ${ }^{1}$ a simpler-tofabricate two-dimensional (2-D) periodic structure may also introduce a large suppression of spontaneous emission. ${ }^{2}$

By introducing a defect in a photonic crystal, a microcavity may be formed with one or more modes strongly localized. These modes are of high interest for realization of lowthreshold lasers. ${ }^{3}$ Such a photonic crystal microcavity is shown in Fig. 1, where the dotted circles represent holes in a GaAs substrate. A microcavity has been introduced by decreasing

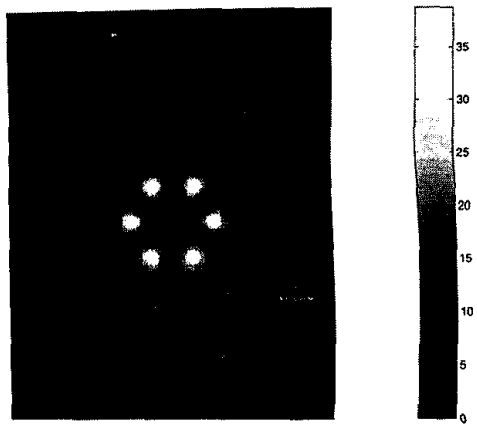

JFA2 Fig. 1. The dotted circles arranged on a triangular lattice represent holes in a GaAs substrate. A defect has been introduced by decreasing the size of an air-hole. The amplitude of the electric field squared is shown for a mode localized to the defect. The center-to-center hole spacing relative to the free-space wavelength is 0.373 .

the size of a single air-hole in the otherwise perfect periodic structure. The amplitude of the electric field squared is also shown for a strongly localized mode. The figures in this text have all been calculated using a planewave method. ${ }^{4}$

Using a semiclassical approach, the change in the rate of spontaneous emission introduced by a photonic crystal is modelled through the concept of the positiondependent density-of-states. The emitters are modelled as electric dipoles. Spontaneous emission is modelled as emission stimulated by vacuum fluctuations in the classically treated electromagnetic modes.

The density-of-states at a given frequency is essentially obtained by counting modes within a narrow frequency interval and normalizing. Position dependence is introduced by further weighting the contribution from each mode with the amplitude of the electric field squared, where the amplitude has been normalized so that the total energy of the mode is unity. This leads to a more correct model for the rate of spontaneous emission compared to using the position-independent density-of-states only. ${ }^{2}$

The position-dependent density-of-states has been calculated for the full periodic structure shown in Fig. 1 with no defect. To the best of our knowledge this is the first calculation of the position-dependent density-of-states for 2-D photonic crystals.

The result is shown in Fig. 2 for a GaAs region mid between two air-holes and in the center of an air-hole in the full periodic photonic crystal (two extremes chosen to illustrate strong position dependence). A similar calculation for homogeneous GaAs is also shown. For the normalized frequency 0.373 corresponding to the mode shown in Fig. 1 (wavelength $=800 \mathrm{~nm}$ and center-to-center hole spacing $=300 \mathrm{~nm}$ ) the curves for homogeneous GaAs and GaAs in the photonic crystal differ by a factor of 1.8. This factor corresponds to a possible 45 percent suppression of spontaneous emission in the GaAs photonic crystal structure. This leads to the conclusion that strong suppression of spontaneous emission in 2-D photonic crystals is feasible, having major implications on future realization of low-threshold lasers. Further analysis of the 


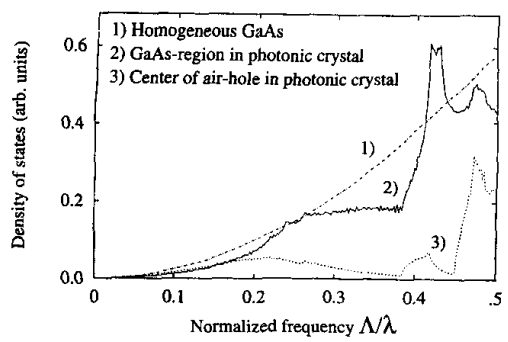

JFA2 Fig. 2. The three-dimensional positiondependent density-of-states is shown for homogeneous GaAs as curve 1) along with the corresponding calculation for two positions in the full periodic photonic crystal characterized by holes having a filling fraction of 0.67 in a GaAssubstrate. Curve 2) represents a GaAs region mid between two air-holes in the photonic crystal, whereas curve 3 ) represents the center of an airhole in the photonic crystal. $\Delta$ is the center-tocenter hole spacing and $\lambda$ is the free-space wavelength.

photonic crystal microcavity will be presented at the conference.

1. E. Yablonovitch. Inhibited spontaneous emission in solid-state physics and electronics. Physical Review Letters, 58(20), 2059-62, May 1987.

2. T. Søndergaard, J. Broeng, A. Bjarklev, K. Dridi, and S.E. Barkou. Suppression of spontaneous emission for a twodimensional honeycomb photonic band gap structure estimated using a new effective-index model. IEEE Journal of Quantum Electronics. 34(12), Dec. 1998.

3. O. Painter, J. Vuckovic, and A. Scherer. Defect modes of a two-dimensional photonic crystal in an optically thin dielectric slab. Journal of the Optical Society of America $B, 1999$. In press.

4. R.D. Meade, A.M. Rappe, K.D. Brommer J.D. Joannopoulos, and O.L. Alerhand. Accurate theoretical analysis of photonic band-gap materials. Physical Review B, 48(11), 84347 Sept. 1993.

Properties of photonic lattices created using arrays of coupled vertical cavlty surface emitting lasers

Harald Pier, Claude-Albert Berseth, Eli Kapon, Michael Moser, ${ }^{*}$ Tal Fishman, ${ }^{* *}$ Amos Hardy, ${ }^{* *}$ Department of Physics, Swiss Federal Institute of Technology (EPFL), Lausanne, CH-1008 Switzerland; E-mail: pier@epfl.ch

Structures with a periodically modulated refractive index are frequently discussed in the context of the possibility of forming a "photonic bandgap". These would be useful for many applications in optoelectronics, e.g. ultra-low threshold lasers, more efficient light emitting diodes or novel waveguides. ${ }^{1}$ Another class of photonic lattices relies on the analogy of the bound photon states in arrays of microcavities to electronic states in a lattice of atoms. Recently, this inspired the realization of photonic molecules ${ }^{2}$ and photonic lattices con-

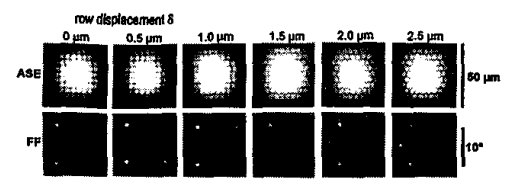

JFA3 Fig. 1. Experimental data. By shifting adjacent rows, strained photonic lattices can be realized (amplified spontaneous emission patterns, ASE). Beyond a critical strain value, the mode undergoes a phase transition, resulting in an abrupt change of the far-field pattern (FF).

structed using arrays of coupled microcavities. Such periodic arrangements of bound photon modes have been used to realize disordered and-lately-strained photonic lattices.

Experimentally, our photonic lattices are defined by metal-patterning highly uniform VCSEL-wafers. Excellent lateral uniformity, required for large coupled lattices, is demonstrated by the small (less than $0.2 \%$ ) variations of the cavity resonance in the reflectivity spectrum measured across the wafer. Lasing only occurs at the locations of high-reflectivity gold pixels placed on a low-reflectivity $\left(R_{b}\right)$ chromium-covered background. Using this approach, the design of the photonic lattices is extremely flexible and is limited only by the lithographic resolution. Typical pixel sizes are $4-5 \mu \mathrm{m}$, and spacings between pixels can be as small as $1 \mu \mathrm{m}$.

The lasing modes of these photonic modes are observed close to threshold. The structures are analyzed theoretically using a coupled resonator model employing the patterned reflectivity of the composite metal-dielectric mirror. ${ }^{3}$ As an example, consider the series of photonic lattices incorporating alternating shear strain shown in Fig. 1. ${ }^{4}$ These strained structures are made by shifting every other row of a $10 \times 10$ coupled-array by $\delta$, as illustrated by the amplified spontaneous emission (ASE) patterns measured below threshold. Above threshold, we find that the near-field (NF) patterns virtually locks to the unstrained lattice patterns up to a critical displacement $\delta \cong$ $1.0 \mu \mathrm{m}$, above which the lattice mode undergoes a phase transition to a hexagonal-like lattice mode. This abrupt switching is evidenced by the measured far-field (FF) of the corresponding lattices (see Fig. 1). The peculiar multi-lobed far-fields are a result of the out-ofphase coupling of adjacent cavities for the mode with the largest optical gain.

The model calculations well reproduce the observed features, as shown in Fig. 2. In particular, the phase transition is found to occur abruptly at $\delta \cong 1.1 \mu \mathrm{m}$ for a background reflectivity $R_{b}=0.985$.

The switching behavior of the lasing mode is further illustrated in Fig. 3, which compares the calculated and measured displacement in the peak at each pixel near the center of the lattice versus the row displacement $\delta$. It can be seen that the phase transition point can be tuned by properly selecting $R_{b}$ (Fig. 3 , right plot).

Similar studies were carried out with tapered lattices, superlattice structures, and ir regular lattices, varying the coupling and the position of the lattice points. Several interesting phenomena have been observed (and will

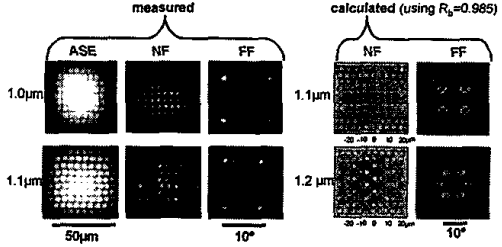

IFA3 Fig. 2. Amplified spontaneous emission pattern (ASE), near- (NF) and far-fields (FF) of lattices close to the transition point at $1.1 \mu \mathrm{m}$ (left). Using the correct value for the reflectivity contrast, this situation can be modeled very accurately (right).

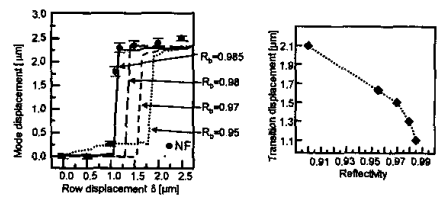

JFA3 Fig. 3. Left: In the experiment, the phase transition occurs between 1.0 and $1.1 \mu \mathrm{m}$ shift (circles, NF). In the model, the background reflectivity $R_{b}$ can be used to tune the switching point. The best fit to the experiment is obtained for $R_{b}=$ 0.985 , which is close to the actual value. Right: Calculated dependence of transition point on reflectivity.

be presented), ranging from decoupling of separated sublattices to injection-level dependent responses that could be useful for beamswitching applications. These studies provide insight into the photonic band structure of two-dimensional photonic crystals.

${ }^{*}$ Optoelectronic Devices, CSEM Zurich, Badenerstrasse 569, 8048 Zurich, Switzerland; E-mail: mmoser@csem.ch

**Tel Aviv University, Department of Electrical Eng.-Physical Electronics, Tel-Aviv 69978, Israel; E-mail: talf@eng.tau.ac.il

1. John D. Joannopoulos, P.R. Villeneuve and S. Fan, Nature 386, 143-149 (1997).

2. M. Bayer et al., Phys. Rev. Lett. 81, 25822585 (1998).

3. T. Fishman, A.A. Hardy, and E. Kapon, IEEE Journal of Quantum Electronics 33, 1756-1762 (1997).

4. H. Pier, E. Kapon, and M. Moser, CLEO Europe, Glasgow, Scotland, 1998, p. CThI1 (invited paper).

\section{JFA4}

9:00 am

Temperature dependence of lasing characteristics for $1.3 \mu \mathrm{m}$ GaAs-based quantum dot lasers

D.L. Huffaker, O. Shchekin, G. Park, Z.Z. Zou, D.G. Deppe, Microelectronics Research Center, The University of Texas at Austin, 10100 Burnet Road, Bldg. 160, M.S. R9900, Austin, Texas 78758 USA; E-mail: dhuf@mail.utexas.edu

Recently ground-state $1.3 \mu \mathrm{m}$ wavelength lasing has been demonstrated at room temperature from GaAs-based uncoated heterostructure lasers using an InGaAs quantum dot (QD) 